\title{
МНОГОКРИТЕРИАЛЬНАЯ ЗАДАЧА ОПТИМИЗАЦИИ ОБЛИКА СПЕЦИАЛЬНОЙ КОЛЕСНОЙ ТЕХНИКИ
}

\section{MULTI-CRITERIA PROBLEM OF OPTIMIZING THE APPEARANCE OF SPECIAL WHEELED VEHICLES}

\section{A. Amosov \\ V. Golikov}

Summary. This paper is an attempt to perform the process of simultaneous optimization of two or more target functions in a given definition area.

The task of multi-criteria optimization is to find a vector of target variables that satisfies the imposed restrictions and optimizes a vector function whose elements correspond to the target functions. These functions form a mathematical description of the satisfaction criterion and are usually mutually conflicting.

This problem occurs in many fields of science and its solution can be applied to many complex mechanical units and devices. Attempts to adapt existing equipment for operation in specific conditions do not allow us to solve the tasks set. The lack of scientific-based methods for forming the technical appearance and forecasting the future values of the defining parameters hinders the development of this class of machines.

Keywords: unit, special transport, design, layout, technical appearance, creation, mathematical model, criteria optimization.

\author{
Амосов Алексей Германович \\ Ассистент, Московский авиационный институт \\ (национальный исследовательский университет) \\ lamosov@yandex.ru \\ Голиков Владислав Андреевич \\ Ассистент, Московский авиационный институт \\ (национальный исследовательский университет) \\ fordik08@mail.ru
}

Аннотация. Данная работа - попытка совершения процесса одновременной оптимизации двух или более целевых функций в заданной области определения.

Задача многокритериальной оптимизации состоит в поиске вектора целевых переменных, удовлетворяющего наложенным ограничениям и оптимизирующего векторную функцию, элементы которой соответствуют целевым функциям. Эти функции образуют математическое описание критерия удовлетворительности и, как правило, взаимно конфликтуют.

Эта проблема встречается во многих областях науки и ее решение может быть применимо к многим сложным механическим узлам и устройствам. Попытки приспособить существующую технику для эксплуатации в специфических условиях не позволяют решать поставленных задач. Отсутствие научно-обоснованных методов формирования технического облика и прогнозирования перспективных значений определяющих параметров сдерживает развитие этого класса машин.

Ключевые слова: агрегат, специальный транспорт, проектирование, компоновка, технический облик, создание, математическая модель, критериальная оптимизация.

тирования, снижение материальных и временных затрат за счет широкого использования современных методов математического моделирования и средств машинной графики при компоновке подъемных механизмов, грузовых платформ, силовых установок, колесного (гусеничного) хода и других систем (рис. 1).

\section{Методика исследования}

Объектом исследования является специальный агрегат. Предметом исследования является процесс компоновки его оборудования. Декомпозиция задач, разработка моделей и алгоритмов базируются на принципах системного подхода. Выявление рациональных конструктивно-компоновочных решений осуществлено на основе моделирования с помощью формально-эвристических процедур. Математическая задача отыскания 

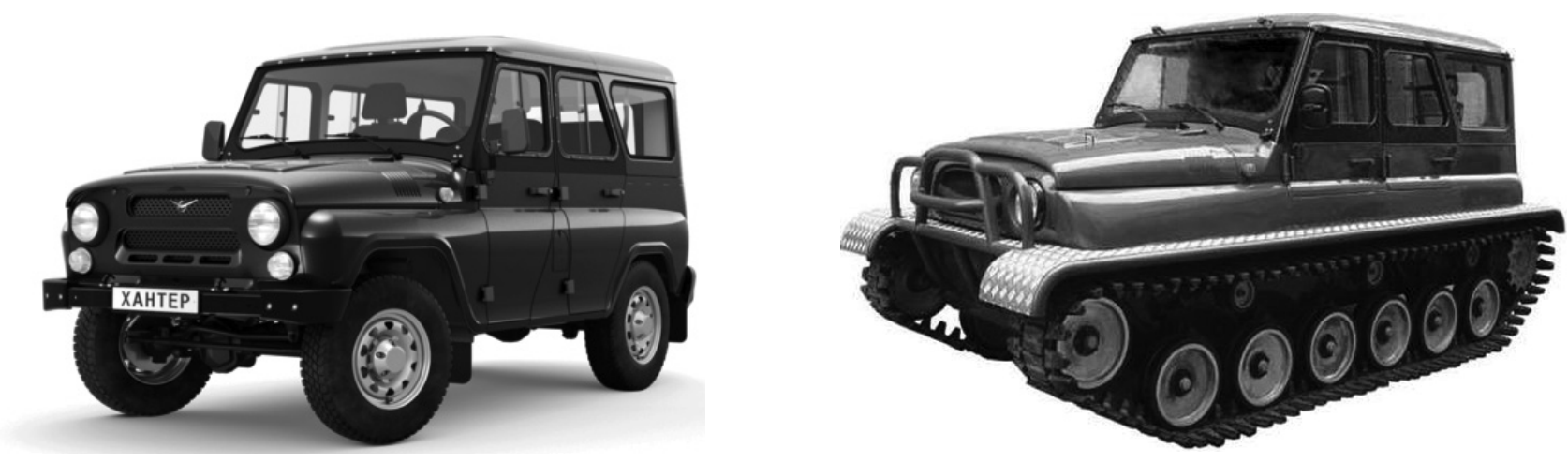

Рис. 1. УАЗ на колесном и гусеничном ходу

рациональных значений параметров поставлена как задача многокритериальной оптимизации.

Технический уровень продукции - относительная характеристика качества продукции, основанная на сопоставлении значений показателей, характеризующих техническое совершенство оцениваемой продукции с базовыми значениями соответствующих показателей [1]. Под техническим обликом будем понимать комплексную качественную и количественную характеристику объекта проектирования, отражающую наиболее общие и значимые признаки рассматриваемого объекта [2].

Технический облик специальных агрегатов формируется в процессе разработки технического предложения [3]. Этот этап играет особую роль в процессе проектирования всей машины, являясь одним из этапов научно-исследовательской работы и занимает положение перед разработкой технического задания на эскизное проектирование. Цель процесса формирования технического облика - обоснование количественных значений основных определяющих параметров проектируемого объекта.

В процессе формирования облика агрегатов принимается более $70 \%$ решений по проекту [3], и от качества этих решений зависит возможность создания объекта в заданные сроки при ограниченном финансировании программы.

Исходными данными для формирования технического облика специальных агрегатов являются результаты анализа [3]:

- предполагаемых областей и условий использования объекта исходя из главного предназначения;

- современных и перспективных требований к эксплуатационным свойствам рассматриваемого класса агрегатов;
- конструктивных решений возможных прототипов и основных тенденций развития мирового машиностроения по агрегатам соответствующего класса.

В настоящее время неизвестны алгоритмы прямого оптимального синтеза сложных технических объектов, и их разработка осуществляется многократным повторением анализа различных вариантов проектных альтернатив. Создание новых агрегатов специального назначения в этом отношении не является исключением.

В большинстве случаев решения задач формирования технического облика речь идет о процессах, не содержащих неопределенностей. Большинство реальных инженерных задач содержит в том или ином виде неопределенности [2], связанные с нечеткостью формулировок целей и задач, размытыми границами ограничений и т.п. Формирование технического облика семейства специальных агрегатов представляет собой решение многопараметрической многокритериальной оптимизационной задачи с неполной информацией об объектах, что связано с отсутствием детальной конструкторской проработки узлов. Однако, из-за наличия концептуальных и методических трудностей в настоящее время не существует единого методологического подхода к решению таких задач.

В литературе есть примеры решения вопросов оптимизации параметров конструкции на этапе проектирования при создании автомобильной техники. Например, вопросам оптимизации автомобилей многоцелевого назначения посвящена работа [2], транспортных средств особо большой грузоподъемности посвящен раздел в книге [3], подходы к оптимальному проектированию грузовых автомобилей рассмотрены в работе [4]. Технико-экономическим анализом сложных агрегатов, что также можно отнести к вопросам оптимизации, занимались авторы работ $[5,6]$. 
Следует отметить, что вопросы оптимизации конструкции и вопросы оценки технического уровня объектов перекликаются между собой и при их решении зачастую используются одинаковые методы.

В последние десятилетия в нашей стране и за рубежом в самых различных отраслях производства разработаны и продолжают разрабатываться многочисленные методики по управлению качеством выпускаемой продукции на стадии проектирования, позволяющие обеспечивать получение изделий с оптимальными параметрами по критерию «цена - качество» [2].

Под качеством продукции будем понимать совокупность свойств продукции, обеспечивающий ее пригодность удовлетворять определенные потребности в соответствии с ее назначением [7].

\section{Математическая молель}

Анализ структуры процедур формирования облика агрегатов, показывает, что отыскание вектора опре-

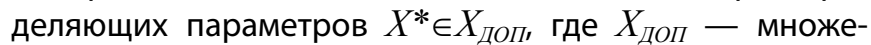
ство допустимых вариантов проекта является сложной многокритериальной задачей. Её в общем виде можно сформулировать так: определить вектор определяющих параметров $X^{*}$, состоящий из элементов, которым соответствует минимальное значение целевой функции $\mathrm{F}(\mathrm{x}$; u), связывающей эти параметры и характеристики проектов на множестве ограничений.

Решение данной задачи как задачи математического моделирования не всегда приводит к успеху из-за значительной размерности вектора определяющих пара-

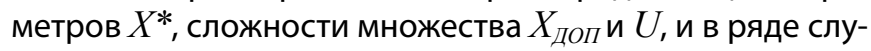
чаев большого количества времени, необходимого для вычисления значений критерия $\mathrm{F}(\mathrm{x} ; \mathrm{u})$.

С целью упрощения задачи используется следующий подход: эффективность агрегатов можно оценить по значениям некоторого набора частных критериев, определяющих концепцию разрабатываемой модели [10].

Исходя из данного подхода математическая постановка задачи, как задачи многокритериальной оптимизации, в общем виде будет представлена следующим образом:

$$
\begin{gathered}
\mathrm{X}^{*}=\underset{\mathrm{x} \in \mathrm{X}}{\operatorname{Arg}} \\
\mathrm{Min} \mathrm{F}(\mathrm{x} ; \mathrm{u}) .
\end{gathered}
$$

$X=X\left(N_{C E \Psi} ; N_{K P} ; C ; K ; T\right)-$ вектор определяющих параметров:
$X=X\left(X_{1} ; X_{2} ; X_{3} ; X_{4} ; X_{5}\right)$.

где $G 1$ - грузоподъемность агрегата в целом;

$G 2$ - грузоподъемность стрелы (подъемного механизма);

$C$ - параметр, характеризующий соотношение полезной нагрузки и полной массы;

$K$ - величина вылета стрелы или высоты подъема подъемного механизма;

$T$ - тип используемого подъемного механизма.

$U=U\left(N_{K Р / Б} ; N_{A . B} ; V_{\text {ПАС }} ; L_{\Phi} ; H_{\Pi}\right)$ - вектор ограничений:

где $N_{K Р / Б}$ - ограничение по грузоподъемности;

$N_{A . B}$ - ограничение по массе перевозимого груза;

$V_{\text {ПАС }}$ - ограничение по высоте вылета стрелы (подъема захвата);

$L$ - ограничение по длине агрегата в целом;

$H_{M}$ - ограничение по полной массе агрегата.

$F=F\left(S 1 ; M_{\Gamma}\right)$ - вектор целевых функций:

где $S 1$ - площадь грузовой платформы;

$$
\mathrm{S} 1=\iint \mathrm{f}(\mathrm{x}, \mathrm{y}) \mathrm{dxdy}
$$

$M_{\Gamma}$ - максимальная масса поднимаемого и перевозимого груза.

Реализация поставленной задачи требует разработки соответствующих математических моделей, включающих в себя методы экспертных оценок. При этом основным математическим аппаратом формализации представления и обработки экспертных оценок и высказываний является теория нечетких множеств [8]. Применение аппарата нечеткого множества - это попытка математической формализации нечетких (экспертных) оценок в виде лингвистически поименованных функций для построения моделей обработки этих оценок как композиции указанных функций, имеющих простую лингвистическую интерпретацию. В результате появляется возможность для конечного пользователя оперировать естественными предметно-ориентированными лингвистическими термами, представляемыми на уровне компьютерных вычислений в виде чисел. Такой подход дает приближенные, но в то же время качественные способы описания поведения сложных и плохо определенных организационно-технических систем. Теоретические же основания данного подхода вполне точны и строги в математическом смысле и не являются сами по себе источником неопределенности. В каждом конкретном случае степень точности решения может быть согласована с требованиями задачи. 


\section{ОбсужАения}

Как было показано выше, определяющим условием создания оптимальной конструкции специальных агрегатов является рациональная компоновка подъемного механизма и грузовой платформы с точки зрения оптимального размещения оборудования. Данное обстоятельство требует разработки соответствующих моделей, методик, алгоритмов и программ и самостоятельной системы, по формированию их облика исходя из его компоновки.

Установлено, что непременными компонентами решения любой творческой задачи являются: оперативные элементы, стратегия и статическая система. В процессе решения задачи проектирования агрегатов, названные компоненты могут быть интерпретированы следующим образом [9]:

- оперативные элементы - функциональные элементы, входящие в состав агрегата и обеспечивающие выполнение им заданной задачи (стрела, система подъема стрелы, грузовая платформа, силовая установка и т.п.).

- стратегия - система правил, позволяющая построить из набора функциональных элементов работоспособный вариант компоновки агрегата и оптимизировать его по некоторому критерию;

- статическая система - особым образом формализованное пространство, позволяющее размещать, координировать и перемещать в нем функциональные элементы [12].

Как было показано ранее, определяющим условием создания агрегатов является его рациональная компоновка относительно всех координатных осей с точки зрения получения стабильных показателей удовлетворяющих потенциальным возможностям выбранного типа подъемного механизма (стрелы). Эти особенности не позволяют в полной мере применить изложенные во введении подходы к автоматизированному проектированию и требуют модификации процесса проектирования. Решение данной задачи требует разработки соответствующих моделей, методик, алгоритмов и программного комплекса, позволяющего производить оценку определяющих параметров на ранних этапах проектирования [11].

\section{Выво $\triangle ы$}

Для успешного решения задачи необходимо разработать модели для каждого элемента на выбранном уровне, при этом в модели верхних уровней включаются модели нижних уровней. На каждом иерархическом уровне модель представляет собой связи, представленные в виде уравнений, описывающие зависимости между параметрами и характеристиками.

Во-первых, это математические модели расчёта геометрических, массовых и иных характеристик компонуемых элементов, а, во-вторых, структурно-параметрические модели подъемного механизма (стрелы).

Геометрические модели описывают отношения между параметрами агрегатов и его составляющих, характеристиками их форм и размеров. Они определяют компоновочные объемы, и допустимые диапазоны компоновки. Данные этих моделей являются входной информацией для весовых, прочностных и иных расчётов, компоновки, разработки технологических процессов и т.д.

Массовые модели обеспечивают расчёт массы отдельных элементов и в целом на всех уровнях проектирования. В основу моделей положены связи между геометрией, нагрузками, особенностями конструктивно-силовой схемы, оборудованием, силовой установки, топливом и полезной нагрузкой. Следовательно, можно представить, что в основе массовых моделей лежат геометрические критерии, которые являются, по сути, параметрами массовых моделей.

Для оценки вариантов проектно-конструкторских решений используется эффективностная модель, позволяющая оценить полученное проектно-конструкторское решение. Модель отражает связи между параметрами отдельных элементов и агрегата в целом, с частными и общими критериями эффективности рассматриваемыми в работе.

При разработке новых и использовании известных моделей принято, что отклонение характеристик физических и математических моделей должно быть не более $\pm 5 \%$.

\section{ЛИТЕРАТУРА}

1. ГОСТ 15467-79. Управление качеством продукции. Основные понятия, термины и определения.—- М.: Госстандарт СССР, 1987. - 29 с.

2. Плиев И. А. Автомобили многоцелевого назначения. Формирование технического облика АМН в составе семейств: Монография.—М.: МГИу, 2011.—262 с.

3. Белоусов Б.Н., Попов С. Д. Колесные транспортные средства особо большой грузоподъемности. Конструкция. Теория. Расчет. / Под общ. ред. Б. Н. Белоусова.- М.: Изд-во МГТУ им. Н. Э. Баумана, 2006. - 728 с.

4. Дьяков И. Ф. Оптимальное проектирование грузовых автомобилей. — Саратов: Издательство Саратовского университета, 1989.— 128 с. 
5. Ипатов А. А. Формирование эксплуатационно-экономических требований к перспективным моделям грузовых автомобилей.- М.: ЗАО «Издательство «Экономика», 2003.- 236 с.

6. Ипатов М. И. Технико-экономический анализ проектируемых автомобилей._- М.: Машиностроение, 1982.— 272 с.

7. ГОСТ 2.116-84. Карта технического уровня и качества продукции. Межгосударственный стандарт.—М.: Госстандарт СССР, 1984.16 с.

8. BellmanR.E., Zadeh L. A. Decision-Makingin Fuzzy Environment // Management Science. — vol. 17. — 1970.— No4.—P. 141-160.

9. Вахидов У.Ш., Беляков В. В., Молев Ю. И. Транспортно-технологические проблемы Северного Кавказа: научное издание — Нижегород. гос. техн. университет им. Р. Е. Алексеева.—Нижний Новгород, 2009.— 330 с.

10. Методика оценки уровня качества продукции с помощью комплексных показателей и индексов. - М.: Издательство стандартов, 1974.

11. Ротштейн А.П., Штовба С.Д. Нечеткий многокритериальный анализ вариантов с применением парных сравнений // Известия РАН. Теория и системы управления.-2001.- №3.-C. 150-154.

12. Калимулин М. Р. Анализ процесса формирования технического облика особо лёгких высокоподвижных колёсных транспортных средств для горных условий эксплуатации // Наука и образование.— 2012.—№ 11.—c. 125

(c) Амосов Алексей Германович ( lamosov@yandex.ru ), Голиков Владислав Андреевич ( fordik08@mail.ru ).

Журнал «Современная наука: актуальные проблемы теории и практики»

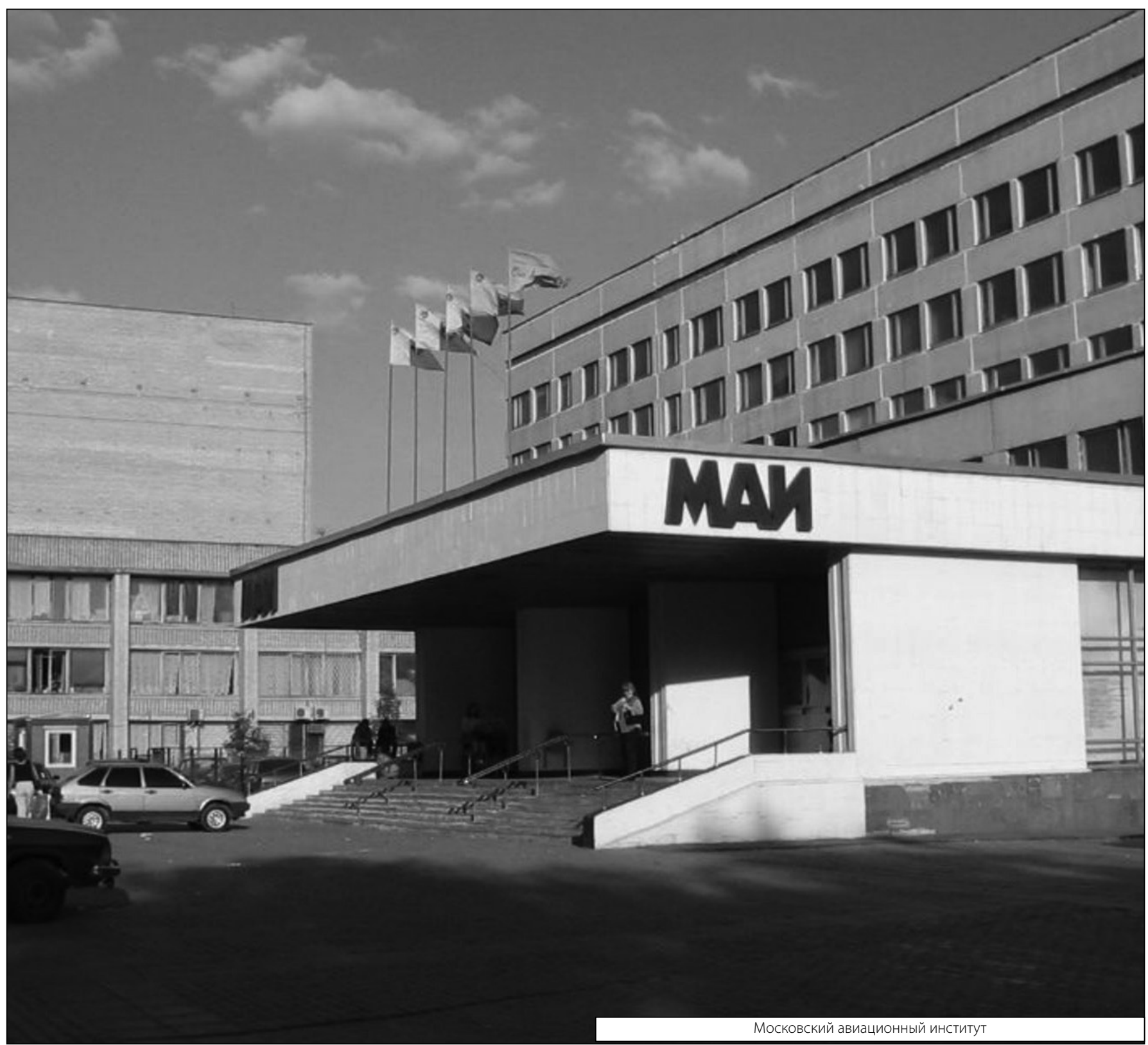

